\title{
Integral Majorization Theorem for Invex Functions
}

\author{
M. Adil Khan, ${ }^{1}$ Adem Kılıçman, ${ }^{2}$ and N. Rehman ${ }^{3}$ \\ ${ }^{1}$ Department of Mathematics, University of Peshawar, Peshawar 25000, Pakistan \\ ${ }^{2}$ Department of Mathematics and Institute of Mathematical Research, Universiti Putra Malaysia (UPM), \\ 43400 Serdang, Selangor, Malaysia \\ ${ }^{3}$ Department of Mathematics and Statistics, Allama Iqbal Open University, H-8, Islamabad, Pakistan
}

Correspondence should be addressed to Adem Kılıçman; akilic@upm.edu.my

Received 18 December 2013; Accepted 11 February 2014; Published 13 March 2014

Academic Editor: S. D. Purohit

Copyright (C) 2014 M. Adil Khan et al. This is an open access article distributed under the Creative Commons Attribution License, which permits unrestricted use, distribution, and reproduction in any medium, provided the original work is properly cited.

We obtain some general inequalities and establish integral inequalities of the majorization type for invex functions. We give applications to relative invex functions.

\section{Introduction}

Let $x, y:[a, b] \rightarrow \mathbb{R}$ be two decreasing real functions. Then the function $x$ is said to majorize $y$ if

$$
\begin{gathered}
\int_{a}^{s} x(t) d t \geq \int_{a}^{s} y(t) d t \quad \text { for } s \in[a, b], \\
\int_{a}^{b} x(t) d t=\int_{a}^{b} y(t) d t
\end{gathered}
$$

(see [1, p. 417] and [2, p. 324]).

The following result is known as majorization theorem for integrals (see [1, p. 417] and [2, p. 325]).

Theorem 1 (see $[1,2])$. Let $x, y:[a, b] \rightarrow I$ be two decreasing real functions, where $I \subset \mathbb{R}$ is an interval. The function $x$ majorizes $y$ if and only if the inequality

$$
\int_{a}^{b} \phi(y(t)) d t \leq \int_{a}^{b} \phi(x(t)) d t
$$

holds for all continuous convex functions $\phi: I \rightarrow \mathbb{R}$ such that the integrals exist.

Some authors have investigated the weighted versions of (2) (see $[1,3,4])$.

In our main results we will use the following definition of invex function.
Definition 2. Let $\phi: I \rightarrow \mathbb{R}$ be a differentiable function on the interval $I$, and let $\eta: I \times I \rightarrow \mathbb{R}$ be a function of two variables. The function $\phi$ is said to be $\eta$-invex if, for all $x, y \in$ I,

$$
\phi(x) \geq \phi(y)+\phi^{\prime}(y) \eta(x, y) ;
$$

see [5, pp. 1]. $\phi$ is called invex if $\phi$ is $\eta$-invex for some $\eta$.

Clearly, each differentiable convex function $\phi: I \rightarrow \mathbb{R}$ is an $\eta$-invex function with $\eta(x, y)=x-y$ for $x, y \in$ $I$. It is known that a differentiable function is invex if and only if each stationary point is a global minimum point [5]. This fact was the motivation to introduce invex functions in optimization theory [6].

Let $\Theta: \mathbb{R}^{2} \rightarrow \mathbb{R}$ be an arbitrary function vanishing at points of the form $(0, b), b \in \mathbb{R}$. It is easy to verify that if a differentiable function $\phi$ satisfies the condition

$$
\begin{aligned}
& \Theta\left(\phi^{\prime}(y), \eta_{0}(x, y)\right) \geq 0 \\
& \text { implies } \phi(x)-\phi(y) \geq 0 \quad \text { for } x, y \in I
\end{aligned}
$$

for some functions $\eta_{0}$, then $\phi$ is invex.

In particular each pseudoconvex function is invex [5, pp. 3-4]. In fact, it is sufficient to consider $\Theta(a, b)=a b$ for $a, b \in$ $\mathbb{R}$ and $\eta_{0}(x, y)=x-y$ for $x, y \in I$.

In the multidimensional case $(n \geq 1)$, we have the following definition. 
Definition 3. Let $\langle\cdot, \cdot\rangle$ be an inner product on $\mathbb{R}^{n}$. Let $\eta$ : $I^{n} \times I^{n} \rightarrow \mathbb{R}^{n}$ be a function of two variables. One has the following.

(i) A differentiable function $F: I^{n} \rightarrow \mathbb{R}$ is said to be $\eta$-invex if, for all $x, y \in I^{n}$,

$$
F(x)-F(y) \geq\langle\nabla F(y), \eta(x, y)\rangle
$$

where $\nabla$ denotes the gradient [5,pp. 1].

(ii) A differentiable function $F: I^{n} \rightarrow \mathbb{R}$ is said to be $\eta$-pseudo-invex if, for all $x, y \in I^{n}$,

$$
\langle\nabla F(y), \eta(x, y)\rangle \geq 0 \quad \text { implies } F(x)-F(y) \geq 0
$$

(see [6]).

(iii) A differentiable function $F: I^{n} \rightarrow \mathbb{R}$ is said to be $\eta$-quasi-invex if, for all $x, y \in I^{n}$,

$$
F(x)-F(y) \leq 0 \quad \text { implies }\langle\nabla F(y), \eta(x, y)\rangle \leq 0
$$

(see [6]).

A differentiable real function $F: I^{n} \rightarrow \mathbb{R}$ is said to be invex (resp., pseudoinvex, quasi-invex), if $F$ is $\eta$-invex (resp., $\eta$-pseudo-invex, $\eta$-quasi-invex) for some functions $\eta: I^{n} \times$ $I^{n} \rightarrow \mathbb{R}^{n}$.

For applications of invex functions in optimization and mathematical programming, see [5-12] and for some recent results of majorization discrete results for invex functions see [13].

In this paper, we extend integral version of majorization theorem from convex functions to invex ones. We also give some applications to relative invex functions.

\section{Main Results}

In the following theorem we obtain an inequality which we will use in our other results.

Theorem 4. Let $\phi: I \rightarrow \mathbb{R}$ be an $\eta$-invex function on the interval $I$, where $\eta: I \times I \rightarrow \mathbb{R}$ is a continuous function, and let $f, g, p:[a, b] \rightarrow I$ be integrable functions with $p(t) \geq 0$ for $t \in[a, b]$. Then

$$
\begin{gathered}
\int_{a}^{b} p(t) \phi(f(t)) d t-\int_{a}^{b} p(t) \phi(g(t)) d t \\
\geq \int_{a}^{b} p(t) \phi^{\prime}(g(t)) \eta(f(t), g(t)) d t .
\end{gathered}
$$

Proof. If we take $x \rightarrow f(t), y \rightarrow g(t)$ in (3), we obtain

$$
\phi(f(t))-\phi(g(t)) \geq \phi^{\prime}(g(t)) \eta(f(t), g(t)) .
$$

Multiplying (9) by $p(t) \geq 0$ and integrating with respect to $t$, we deduce (8). holds.

The following weighted integral majorization theorem
Theorem 5. Let $\phi: I \rightarrow \mathbb{R}$ be an $\eta$-invex function on the interval $I$, where $\eta: I \times I \rightarrow \mathbb{R}$ is a continuous function, and let $f, g, p:[a, b] \rightarrow I$ be integrable functions with $p(t)>0$ for $t \in[a, b]$. Moreover if $\phi^{\prime}(g(t))$ and $\eta(f(t), g(t))$ are increasing (decreasing) on $[a, b]$ and

$$
\int_{a}^{b} p(t) \eta(f(t), g(t)) d t=0
$$

then

$$
\int_{a}^{b} p(t) \phi(f(t)) d t \geq \int_{a}^{b} p(t) \phi(g(t)) d t .
$$

Proof. We know that the Chebyshev's inequality is

$$
\begin{aligned}
& \frac{1}{\int_{a}^{b} p(t) d t} \int_{a}^{b} p(t) F(t) G(t) d t \\
& \quad \geq \frac{1}{\int_{a}^{b} p(t) d t} \int_{a}^{b} p(t) F(t) d t \frac{1}{\int_{a}^{b} p(t) d t} \int_{a}^{b} p(t) G(t) d t,
\end{aligned}
$$

where $F, G:[a, b] \rightarrow \mathbb{R}$ are the functions of same monotonicity and $p:[a, b] \rightarrow \mathbb{R}^{+}$is any integrable function.

By assumption the functions $\phi^{\prime}(g(t)), \eta(f(t), g(t))$ : $[a, b] \rightarrow \mathbb{R}$ have the same monotonicity. Therefore, by applying Chebyshev's inequality (12) in the right hand side of (8), we have

$$
\begin{gathered}
\int_{a}^{b} p(t) \phi^{\prime}(g(t)) \eta(f(t), g(t)) d t \\
\geq \frac{1}{\int_{a}^{b} p(t) d t} \int_{a}^{b} p(t) \phi^{\prime}(g(t)) d t \\
\quad \times \int_{a}^{b} p(t) \eta(f(t), g(t)) d t .
\end{gathered}
$$

Also (10) holds, so from (8) and (13) we have

$$
\begin{gathered}
\int_{a}^{b} p(t) \phi(f(t)) d t-\int_{a}^{b} p(t) \phi(g(t)) d t \\
\geq \int_{a}^{b} p(t) \phi^{\prime}(g(t)) \eta(f(t), g(t)) d t \\
\geq \frac{1}{\int_{a}^{b} p(t) d t} \int_{a}^{b} p(t) \phi^{\prime}(g(t)) d t \\
\quad \times \int_{a}^{b} p(t) \eta(f(t), g(t)) d t=0 ;
\end{gathered}
$$

then we deduce the desired result (11).

Remark 6. In Theorem 5 the assumption (10) is a strong condition for $p, \eta$. This can be relaxed if a monotonicity property for the $\eta$-invex function $\phi$ is assumed. 
Theorem 7. Let all the assumptions of Theorem 5 hold, but instead of (10) one has the assumption that

$$
\int_{a}^{b} p(t) \eta(f(t), g(t)) d t \geq 0
$$

and $\phi: I \rightarrow \mathbb{R}$ is an $\eta$-invex increasing function on the interval I; then (11) holds.

Proof. The proof is similar to the proof of Theorem 5 but by (15) and monotonicity of $\phi$ we have

$$
\int_{a}^{b} p(t) \phi^{\prime}(g(t)) d t \geq 0, \quad \int_{a}^{b} p(t) \eta(f(t), g(t)) d t \geq 0 .
$$

Using these in (13) we have

$$
\int_{a}^{b} p(t) \phi^{\prime}(g(t)) \eta(f(t), g(t)) d t \geq 0 .
$$

Theorem 8. Let $\phi: I \rightarrow \mathbb{R}$ be an $\eta$-invex function on the interval $I$, where $\eta: I \times I \rightarrow \mathbb{R}$ is a continuous function, and let $f, g, p:[a, b] \rightarrow I$ be integrable functions with $p(t) \geq 0$ for $t \in[a, b]$. One has the following.

(i) If $\phi^{\prime} \circ g$ is increasing on $[a, b], \int_{a}^{x} p(t) \eta(f(t), g(t)) d t \leq$ 0 for all $x \in[a, b]$ and $\int_{a}^{b} p(t) \eta(f(t), g(t)) d t=0$, then

$$
\int_{a}^{b} p(t) \phi(f(t)) d t \geq \int_{a}^{b} p(t) \phi(g(t)) d t
$$

(ii) If $\phi^{\prime} \circ f$ is decreasing on $[a, b], \int_{a}^{x} p(t) \eta(g(t), f(t)) d t \geq$ 0 for all $x \in[a, b]$ and $\int_{a}^{b} p(t) \eta(g(t), f(t)) d t=0$, then

$$
\int_{a}^{b} p(t) \phi(f(t)) d t \leq \int_{a}^{b} p(t) \phi(g(t)) d t
$$

Proof. Let set $G(x)=\int_{a}^{x} p(t) \eta(f(t), g(t)) d t$; then $G(x) \leq 0$ for all $x \in[a, b]$, and $G(a)=G(b)=0$. If $\phi^{\prime} \circ g$ is increasing on $[a, b]$, then

$$
\begin{aligned}
& \int_{a}^{b} p(t) \phi(f(t)) d t-\int_{a}^{b} p(t) \phi(g(t)) d t \\
& \quad \geq \int_{a}^{b} p(t) \phi^{\prime}(g(t)) \eta(f(t), g(t)) d t \\
& \quad=\int_{a}^{b} \phi^{\prime}(g(t)) d G(t) \\
& =\left.\phi^{\prime}(g(t)) G(t)\right|_{a} ^{b}-\int_{a}^{b} G(t) d \phi^{\prime}(f(t)) \\
& \quad=-\int_{a}^{b} G(t) d \phi^{\prime}(g(t)) \geq 0 .
\end{aligned}
$$

This proves (18).
Similarly, setting $F(x)=\int_{a}^{x} p(t) \eta(g(t), f(t)) d t$, then $F(x) \geq 0$ for all $x \in[a, b]$, and $F(a)=F(b)=0$.

Now if $\phi^{\prime} \circ f$ is decreasing on $[a, b]$, then by using Theorem 4 we have

$$
\begin{aligned}
& \int_{a}^{b} p(t) \phi(g(t)) d t-\int_{a}^{b} p(t) \phi(f(t)) d t \\
& \quad \geq \int_{a}^{b} p(t) \phi^{\prime}(f(t)) \eta(g(t), f(t)) d t \\
& \quad=\int_{a}^{b} \phi^{\prime}(f(t)) d F(t) \\
& =\left.\phi^{\prime}(f(t)) F(t)\right|_{a} ^{b}-\int_{a}^{b} F(t) d \phi^{\prime}(f(t)) \\
& =-\int_{a}^{b} F(t) d \phi^{\prime}(f(t)) \geq 0 .
\end{aligned}
$$

This proves (19).

The following extension of majorization theorem for relative invex function can be given.

Theorem 9. Let $f, g, p:[a, b] \rightarrow \mathbb{R}$ be integrable functions with $p$ being positive function. Suppose $\phi, \psi: \mathbb{R} \rightarrow \mathbb{R}$ are such that $\psi$ is a strictly increasing function and $\phi \circ \psi^{-1}$ is $\eta$ invex function on $\mathbb{R}$, where $\eta: \mathbb{R} \times \mathbb{R} \rightarrow \mathbb{R}$ is a continuous function. One has the following.

(i) If $\left(\phi \circ \psi^{-1}\right)^{\prime} \circ \psi \quad{ }^{\prime} g$ and $\eta(\psi(f(t)), \psi(g(t)))$ are increasing (decreasing) on $[a, b]$, and $\int_{a}^{b} p(t) \eta(\psi(f(t)), \psi(g(t))) d t=0$, then

$$
\int_{a}^{b} p(t) \phi(f(t)) d t \geq \int_{a}^{b} p(t) \phi(g(t)) d t .
$$

(ii) If $\left(\phi \circ \psi^{-1}\right)^{\prime} \circ \psi \circ g$ is increasing on $[a, b]$, $\int_{a}^{x} p(t) \eta(\psi(f(t)), \psi(g(t))) d t \leq 0$ for all $x \in[a, b]$, and $\int_{a}^{b} p(t) \eta(\psi(f(t)), \psi(g(t))) d t=0$, then

$$
\int_{a}^{b} p(t) \phi(f(t)) d t \geq \int_{a}^{b} p(t) \phi(g(t)) d t .
$$

(iii) If $\left(\phi \circ \psi^{-1}\right)^{\prime} \circ \psi \circ f$ is decreasing on $[a, b]$, $\int_{a}^{x} p(t) \eta(\psi(g(t)), \psi(f(t))) d t \geq 0$ for all $x \in[a, b]$, and $\int_{a}^{b} p(t) \eta(\psi(g(t)), \psi(f(t))) d t=0$, then

$$
\int_{a}^{b} p(t) \phi(g(t)) d t \leq \int_{a}^{b} p(t) \phi(f(t)) d t .
$$

Proof. The proof of this theorem is similar to the proof of Theorems 5 and 8.

Remark 10. By using $\eta(x, y)=x-y$ in Theorems $4,5,7,8$, and 9 we recover the integral majorization results for convex functions given in $[1,4,14]$. 


\section{Conflict of Interests}

The authors declare that there is no conflict of interests regarding the publication of this paper.

\section{Acknowledgment}

The authors are grateful to the anonymous referees for careful checking of the details and for helpful comments that improved the paper. The authors gratefully acknowledge that this research was partially supported by the University Putra Malaysia under the GP-IBT Grant Scheme having project no. GP-IBT/2013/9420100.

\section{References}

[1] N. S. Barnett, P. Cerone, and S. S. Dragomir, "Majorisation inequalities for Stieltjes integrals," Applied Mathematics Letters, vol. 22, no. 3, pp. 416-421, 2009.

[2] J. E. Pecaric, F. Proschan, and Y. L. Tong, Convex Functions, Partial Orderings and Statistical Applications, Academic Press, New York, NY, USA, 1992.

[3] C. P. Niculescu and F. Popovici, "The extension of majorization inequalities within the framework of relative convexity," Journal of Inequalities in Pure and Applied Mathematics, vol. 7, no. 1, article 27, pp. 1-6, 2006.

[4] J. Pečarić and S. Abramovich, "On new majorization theorems," Rocky Mountain Journal of Mathematics, vol. 27, no. 3, pp. 903911, 1997.

[5] A. Ben-Israel and B. Mond, "What is invexity?" Journal of the Australian Mathematical Society B, vol. 28, no. 1, pp. 1-9, 1986.

[6] M. A. Hanson, "On sufficiency of the Kuhn-Tucker conditions," Journal of Mathematical Analysis and Applications, vol. 80, no. 2, pp. 545-550, 1981.

[7] B. D. Craven, "Duality for generalized convex fractional programs," in Generalized Concavity in Optimization and Economics, S. Schaible and W. T. Ziemba, Eds., pp. 473-489, Academic Press, New York, NY, USA, 1981.

[8] M. A. Noor, "Invex equilibrium problems," Journal of Mathematical Analysis and Applications, vol. 302, no. 2, pp. 463-475, 2005.

[9] S. K. Mishra and G. Giorgi, Invexity and Optimization, Springer, New York, NY, USA, 2008.

[10] S. K. Mishra, J. S. Rautela, and R. P. Pant, "Optimality and duality in complex minimax optimization under generalized $\alpha$ invexity," Journal of Nonlinear and Convex Analysis, vol. 11, no. 2, pp. 357-368, 2010.

[11] S. K. Mishra and N. G. Rueda, "Generalized invexity-type conditions in constrained optimization," Journal of Systems Science and Complexity, vol. 24, no. 2, pp. 394-400, 2011.

[12] S. K. Padhan and C. Nahak, "Second order duality for the variational problems under $\rho$ - $(\eta, \theta)$-invexity," Computers and Mathematics with Applications, vol. 60, no. 12, pp. 3072-3081, 2010.

[13] M. Niezgoda and J. Pečarić, "Hardy-Littlewood-Pólya-type theorems for invex functions," Computers and Mathematics with Applications, vol. 64, no. 4, pp. 518-526, 2012.

[14] L. Maligranda, J. E. Pecaric, and L. E. Persson, "Weighted favard and berwald inequalities," Journal of Mathematical Analysis and Applications, vol. 190, no. 1, pp. 248-262, 1995. 


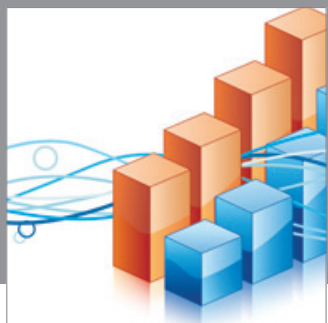

Advances in

Operations Research

mansans

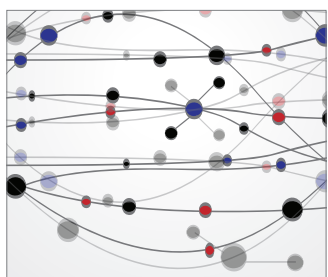

The Scientific World Journal
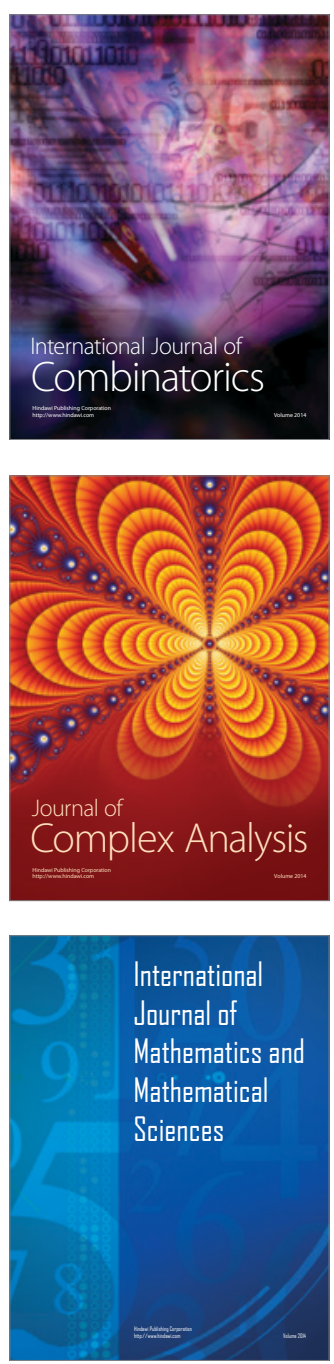
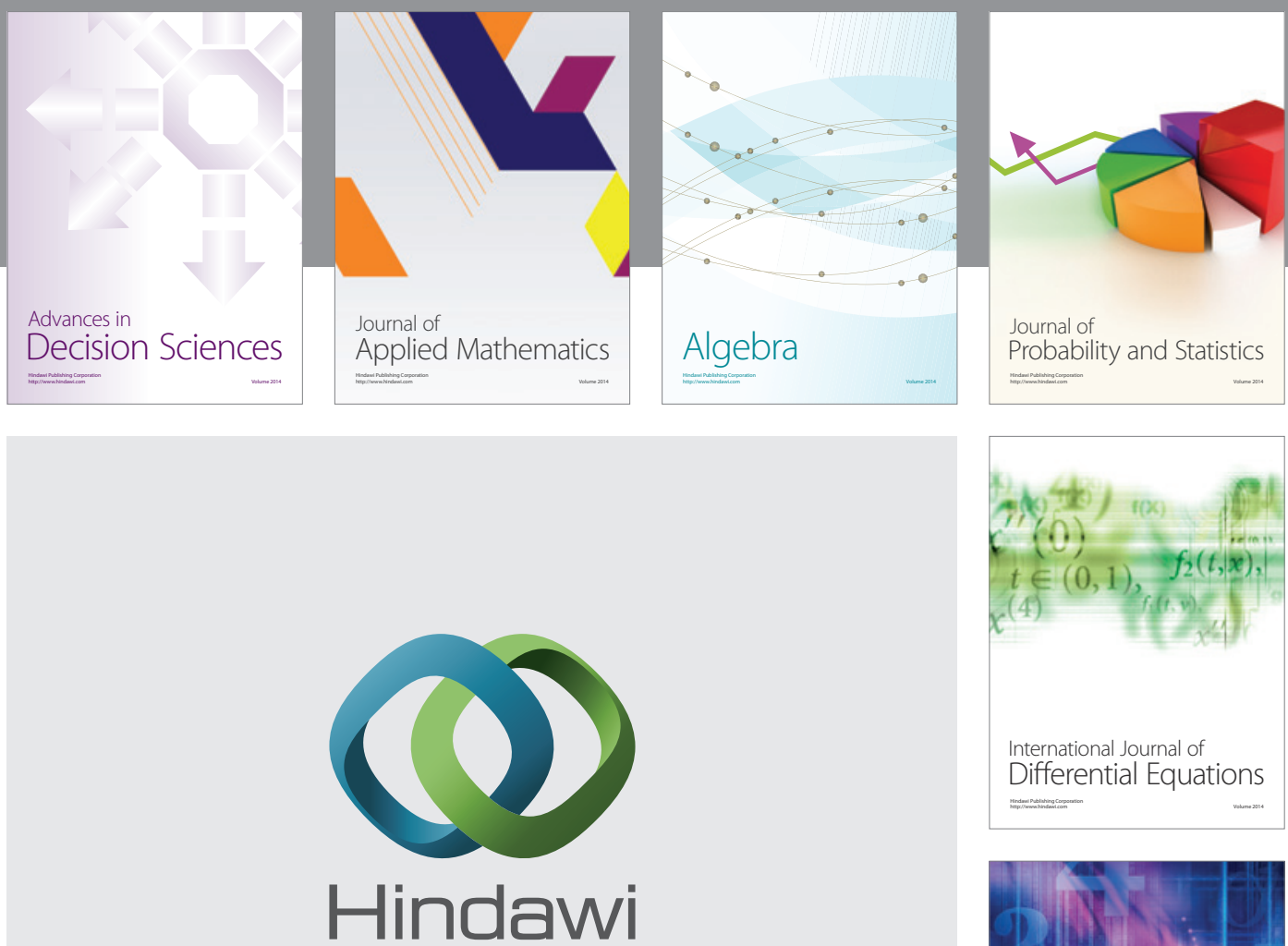

Submit your manuscripts at http://www.hindawi.com
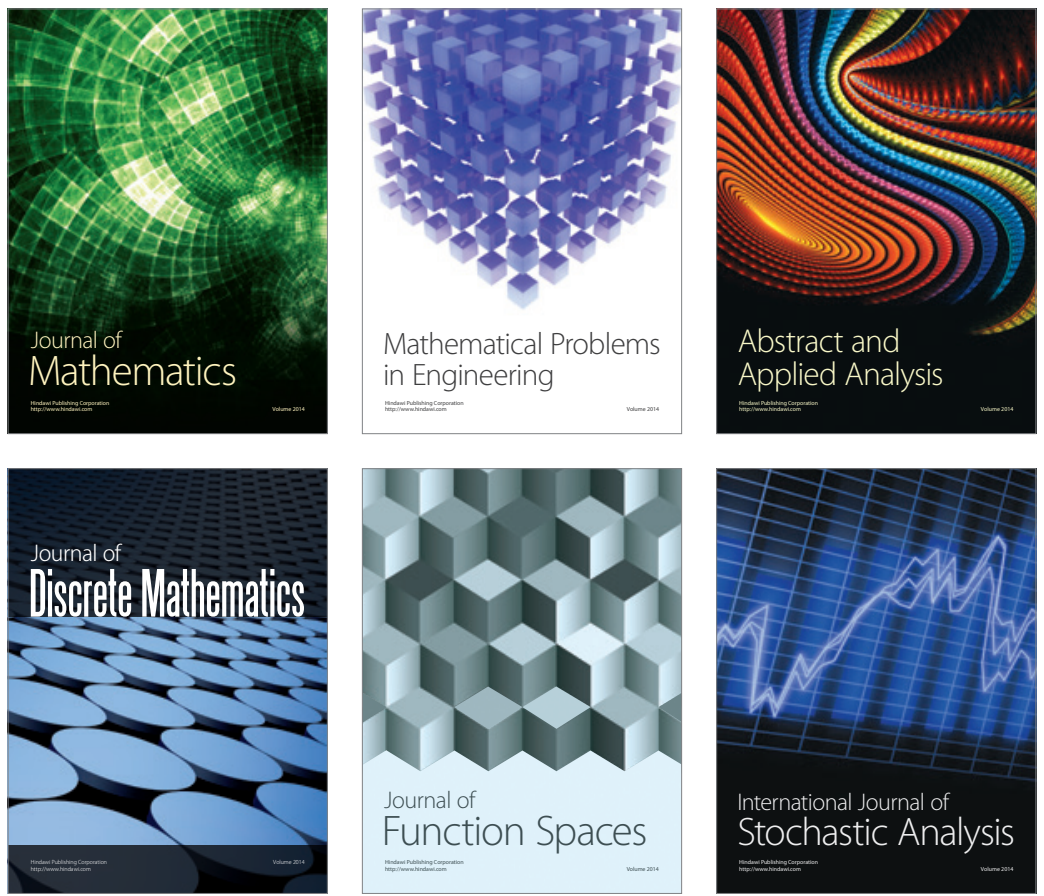

Journal of

Function Spaces

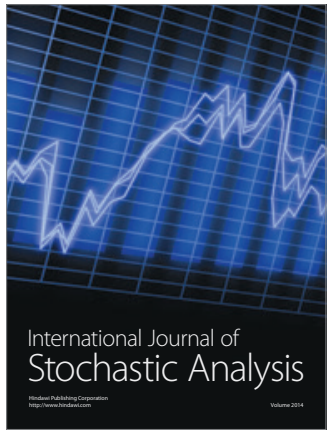

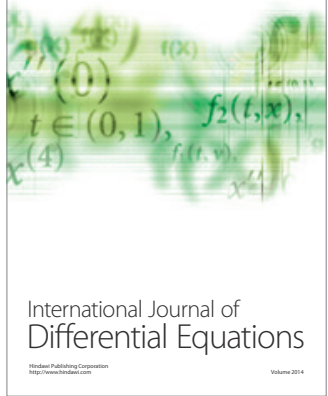
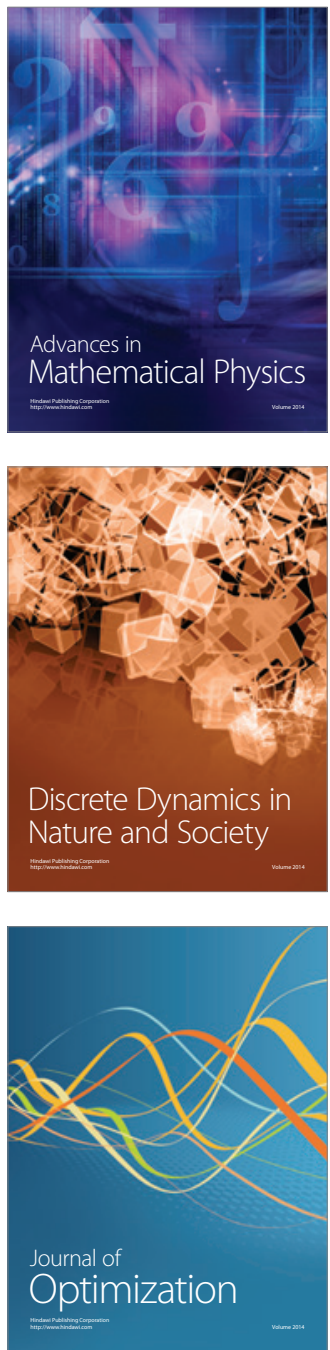